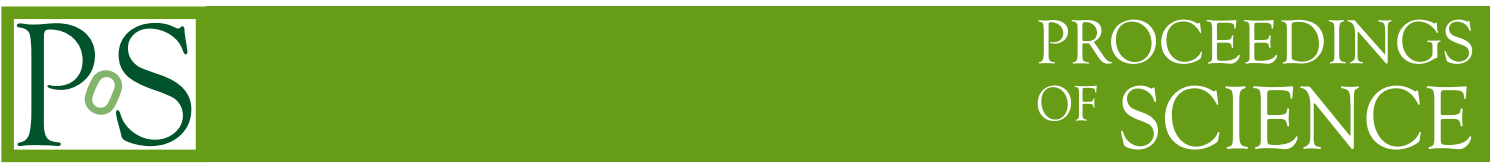

\title{
RCW 86 - A shell-type supernova remnant in TeV gamma-rays
}

\section{Ira Jung-Richardt*}

Friedrich-Alexander University Erlangen-Nürnberg, Department of Physics, Erwin-Rommel-Str.

1, 91058 Erlangen, Germany

E-mail: ira.jung@fau.de

for the H.E.S.S. collaboration

RCW 86 (also known as G 315.4-2.30 or MSH 14-63) is a young supernova remnant about 1800 years old displaying a shell-like structure in the optical, radio, infrared and X-rays regimes with a diameter of about 40'. We will show detailed morphological and spectral studies of the TeV gamma-ray data measured with the High Energy Stereoscopic System (H.E.S.S). These studies reveal for the first time a shell-like structure in this energy range that correlates with the nonthermal X-ray emission ( $2 \mathrm{keV}-5 \mathrm{keV}$ ) observed in the south west region of the remnant. The $\mathrm{TeV}$ gamma-ray spectrum is best described by an exponential cutoff power law. Leptonic and hadronic gamma-ray emission scenarios are probed for RCW 86 in a multi-wavelength approach, whose implications will also be discussed.

The 34th International Cosmic Ray Conference,

30 July- 6 August, 2015

The Hague, The Netherlands

${ }^{*}$ Speaker. 